\section{INSTRUMENTS DE MESURE}

\section{LE TOURNeSOL : APPAREIL POUR LA Mestite DES SECTIONS TPANSVERSALES DES SOUTERRAINS}

Ja levée des scctions fransversales dans les travaux soulerrains est, en général, une opération lente ct souvent fas lidieuse, pour ne pas dire plus. Les méthodes usuelles, quoique conformes aux dounées de la thécrie, n'offent que bien feu de sécurilé en ce qui concerne le degre d'exaclitude qui perul être obtenu, et exigent, de la part de l'opérateur consrinncieus, une lépenes de lemps rarement proporlionnéc ¿ l'importance du travail en Jui-même (*).

Dans cet ordre d'illées, il peut être intéressant pour les lecteurs du foumal de connaître les méthodes el les instruments cmployés à l'étranger et, en parliculier, un appareil spécial réccmment imaginé par un ingénieur américain cmployé aux travaux de l'aquedue de Croton qui alimente la ville de New-York.

Cet instrument consiste principalement en un disque on eercle gradué D fixé à un lube ou ligo de métal mobile en hauleur à l'intéricur d'un autre tube concentrifue, muni fo vis calantes, el placé sur un trépied ordinatere de nivean on

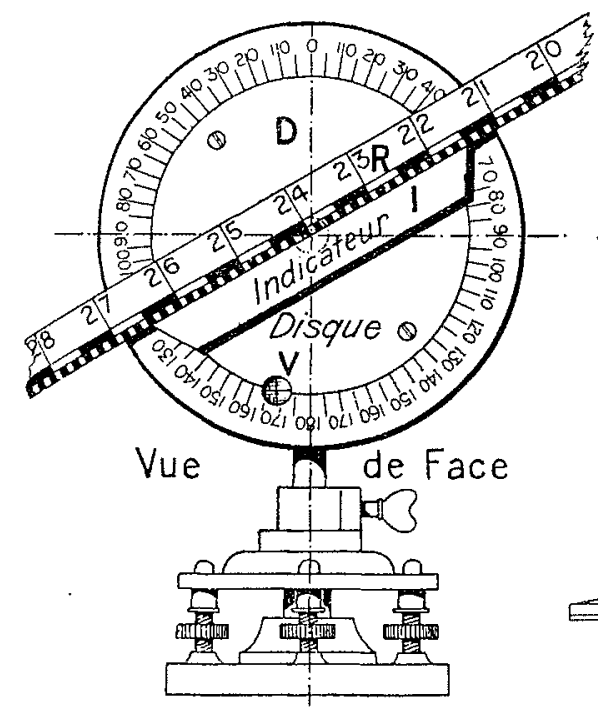

Fig. 1.

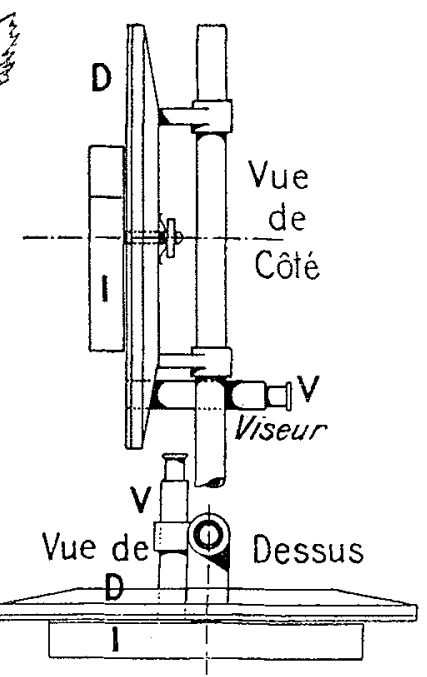

Fig. 2. de théodolite. Le lube porte-disque peut également se mouvoir sur son axe de manièce à présenter la face du disque dans une direclion quelconque.

l'apparence générale de l'instrument monlé sur son trépied rappelle vaguement la forme de la fleur bien connue sous le nom de Tournesol et, faute d'une meilleure dénominalion, nous lui conserverons ce nom dont il a élé baptisé par son invenleur.

la partie antérieure du disque est divisée sur la circonférence en degrés numćrolés de $0^{\circ}$ ì $180^{\circ}$ ì droite di gauche ¿ partir du zéro placé à la partie supérieme du discque.

Au contre de ce disque se meut l'indicateur 1, règle to bois quadrangulate dont la partic on anes mpéricano correspond cactement au diamòtre du cercle, c'est-ì-dire pases exactement par le cenlre du disque. Cel indiraleur, muni d'un pivot traversant le disque, peut se mouvoir sur toule la circonférence du cercle gradué el peul se fixer sur un des diamètres quelennques an moyen de la vis de pression dont est pourvu te pivot ì son extrémité postérieure.

\footnotetext{
(*) Extrait du Journal des Giomètres-Experts.
}

Le tube porle-disque peul êre fixé à une hautcur déterminée au moyen de la vis de pression ; il peut également étre placé dans une posilion exactement verticale au moyen des vis de calage dont est muni le trépied. La ligne o-r 80 du cercle graduć doit the rigoureuscment parallède ì l'ax' du lube porte-disque, de telle manière que lorsque celui-ci est verlical, la dite ligne soit également verticale.

Pour placer le disque dans la direction voulue, on se sorl d'une lunetle spéciale (Viscur) dont lobjectif apparaît en $\mathrm{V}$ sur la vue en dessous el l'oculaire en $\mathrm{V}$ sur li vue de côlé. On peut done situer le disque exactement dans ir meme plan verlical que la section que l'on se propose de lever.

Supposons maintenant l'appareil en station sur ou à peu près sur l'are du souterain el le disque fixí à me haulem

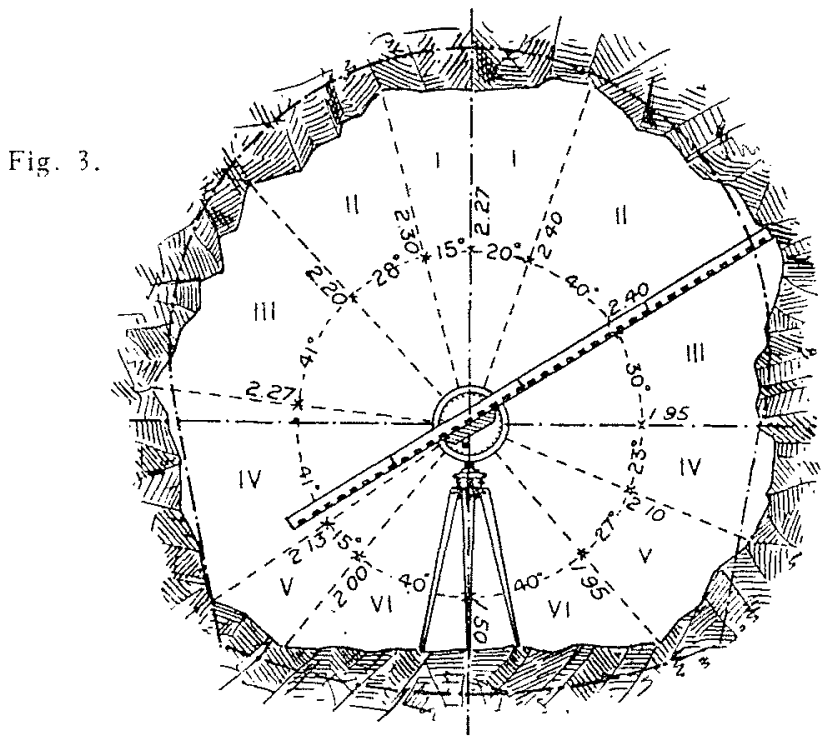

d'cnviron $I^{\mathrm{m}}$ ho au-dessus du sol el placé dans le plan de la seclion transversale. Le centre du disque pourra être considécé comme te pôle d’un syslène de condomécs politires, dont laxe est représenté par la verticale passant par le centre du disque, et dont les angles privent elre mesurís au moyen du cercle graduć.

Plaçons ensuite sur lindicateur une r'ègle graduće (R) quo nous ferons glisser jusquau moment où son extrémilé zéro touchera mo point de la paroi du souterain. La gradualion de la regle qui se trouvera en face du cenlre du disque dí signé sur l'indicalem au moyen d'un index pourvu d'um vernier, marquera la longueur de Ja règle intercopléc que nous pourrons considérer comme un rayon vecleur.

Si nous répétons celle mème opération pour tous les point. du périmètre de la section, en ayant soin de noter exactement l'amplitude de l'angle an centre qui correspond it chaque rayon vecteur, nous amons ainsi obtenu lous Jos éléments nécessaires pour powyoir reporlor a l'échrlle lo profil transversal du soulerrain el calculer mathómaliquement la surface de la section.

le maniement de cet appareil es simple que hous ne aroyons pas nécessaire de pousser plus loin la démonstralion

Le carnel d'observalion est disposé comme un carnol opdinaire de prolils en lravers, la page de gauche élant réservée pour les observations à la gauche de l'axe el la page de droite pour celles à droite.

Chaque page a quate colonnes : dans la premieres'inscrivent les numéros des lriangles formés par Je côté du périmètre ef les deux rayons velenus. On pourrait aussi numéroter les points du périmòre dont la cote a été prise; dans ce cas, il y aurait lien d'ajouler une cinquième colonne. 
Dans la scconde colonne on inscrit les trois facteurs du calcul : les deux rayons vecteurs et l'angle compris. La troisième colonne est réservée aux logarithmes des rayons veclcurs et du sinus de l'angle compris.

Enfin, la quatrième colonne esl destince à recevoir le résultat du calcul de chaque triangle selon la formule bien connue qui permet d'oblenir la surface d'un triangle en fonction de deux colés et de l'angle compris; le nombre qui s'inscrit dans celte colonne est le double de la surface cherchée. Les produits partiels ainsi oblenus s'additionnent et le lolal divisé par deux donnera la vraie superficie de la seclion, qui, dans le cas de la figure 3 est de 13,598 mètrus carrés.

\section{ACADÉMIE DES SGIENCES}

\section{MÉCANIQUE ET ÉLECTRICITÉ}

Sur la " maladie de l'écrouissage " dans l'acier. Note de M. Goorges CharPy, séance du $1 \mathrm{cr}$ août 1910.

$M$. le professeur Cohen a récemment décrit, sous le nom de muladie de l'écrouissage, des observations très intéressantes sur les transformations de l'étain. Un certain nombre de fails, que nous arons obscrvés depuis longtemps dans l'étude des transformations de l'acier, nous paraissent pouvoir ètre utilement rapprochés de ceux qui ont été signalés par M. Cohen.

On sail que l'une des modifications les plus importantes que peuvent subir les mélaux est la cristallisation par recuit, ou plus exactement le développenent progressif des grains cristallins qui constituent un métal, quand on maintient ce métal à une température convenable. En $\mathrm{x} 893\left(^{*}\right)$, nous avons présenté à l'Acalémic les premiers résultals obtenus dans l'élude de ce phénomène, par l'emploi de la micrographie dans le cas du laiton. la cristallisation par recuit de l'acier doux a fait, entre autres ćludes, l'objet d'un important travail de M. Slead, qui a montré, (') 1898 , que lo développement des grains de ferrite dans l'acier doux se faisail avce la plus grande rapidilé, aux températures comprises entre $650^{\circ}$ et $800^{\circ}$. En reprenant l'étude de ce phénomène, cn tenant comple de l'état d'écrouissage du métal, nous avons constaté, of c'est là le point qui nous paraît mériter l'atlention, que, toutes choses égales d'ailleurs, l'écrouissage préalable du métal augmente, dans une proportion considérable, la vitesse de développemenl des grains de ferrite, à une température donnćc. C'est ce que permettent de constater de nombreuses expériences ; nous citerons, en particulier, la suivante :

On prend une barre d'acier doux présentant un grain uniforme ; on la sépare en deux parties, dont l'une est conservée comme témoin, tandis que l'aulre est écrouie par étirage à la filière; les deux fragmenls sont ensuitc recuits simultanément, et maintenus pendant un certain temps à des températures comprises entre $650^{\circ}$ et $800^{\circ}$.

Après refroidissement, on peul, en général, rien qu'en cassant les barres, constater à l'cil nu que le grain est beaucoup plus grossier dans la barre préalablement écrouic que dans l'autre. Si l'écrouissage n'a élé que superficiel, on voit souvent, dans la mème barre, un grain grossier ì la périphérie, tandis que la région centrale présente un grain analogue à celui de la barre non écrouic.

L’examen microscopique permet de préciser le résultal, et de constater que la différence ne réside que dans les dimensions des grains de ferrite. Le rapport des dimensions des grains peut prondre des valeurs considérables, car il semble qu'à une tempéralure convenable, et qui varic avec la nature de l'acier, le développemont des grains de ferrite ne soit pas limité, et qu'on puisse ramener, au bout d'un temps suffisamment long, un bloc de métal à ne plus former qu'un seul grain.

(*) G. Charpy. Influence de la température de recuit sur la structure et les propriétés mécaniques du laiton. (Comptes rendus, I er semestre I 803).
Avec des acicrs doux thès pauves en carbone, el légèrement phosphorcux, le phénomène est extrêmement marqué, et l'on obscrve facilement, après un même recuil, des grains dont les dimensions linéaires varient dans le rapport de $I$ à ro, suivant l'état d'écrouissage préalable.

Ainsi, dans le spécimen que nous présentons à l'Académie, le volume des grains est, en moyenne, mille fois plus grand dans la partie écronie que la partic non écrouie. Ce développement du grain ne modifie pas considérablement, comme l'on sait, les propriétés mécaniques que révèle l'essai de traction ordinaire, mais présente, en revanche, une inlluence énorme sur la fragilité du métal. Le travail spécifique de rupture, ou résilience, tel qu'il est mesuré par l'essai de flexion sur barreaux entaillés, devient pratiquement nul dès que les dimensions des grains atteignent une certaine valeur. Dans cerlains cas, nous avons observé que des barres d'acier doux qui, après étirage à la filière, pouvaient subir une flexion très accentuée sans être détériorées, devenaient, après un recuit aux environs de $650^{\circ}$, assez fragiles pour se casser en tombant à terre. Ainsi, la diminution de mal. léabilité produite dans l'acier doux par l'écrouissage, loin d'ètre toujours atténuée par le recuit, comme on l'admet généralement, peut ètre, au contraire, très fortement exagéréc, lorsque ce recuit a lien dans un certain intervalle de température, qui est, en général, voisin de $650^{\circ}-800^{\circ}$.

Ce phénomène s'observe également sur les aciers écrouis par les procédés les plus divers, fils tréfilés, tôles laminées à froid, aciers martelés ou cmboutis à froid, etc., etc. Si l'on considère le grand nombre d'opérations du travail des métaux qui sont susceplibles de produire un écrouissage, et qui allernent avec des réchauffages, on sera conduit à reconnaître que le phénomène auquel est consacréc cette Note doit intervenir fréquemment pour modifier la qualité des produits métallurgiques et pourrait, sans doute, servir d'explication, dans bien des cas, à des ruplures jugées tout à fait anormales.

Dans certains cas, ce phénomène peut être utilisé comme un véritable révélateur de l'état d'écrouissage d'un mélal. Supposons, par exemple, qu'on produise des empreinles sur un fragment de métal, puis qu'on le polisse de façon à faire disparaître tout relief. Si l'on recuit ce nétal et si, après l'avoir attaqué chimique. ment, on l'examine au microscope, on constate que les grains cristallins sont beancoup plus développés dans les parties qui correspondaient aux creux les plus accentués; la différence de grosseur des grains peut ètre assez marquée pour être nettement visible à l'œil nu.

En résumé, les faits que nous venons de rappeler, et un grand nombre d'autres que nous avons pu observer, nous conduisent donc à conclure que l'écrouissage préalable augmente dans une très forte proportion la vitesse de cristallisation par recuit.

\section{INFORMATIONS DIVERSES}

\section{Usine hydro-électrique du Tage}

On a, depuis peu, mis en service une usine hydro-électrique qui utilise les eaux du Tage, près de Pastrana, et envoie de l'énergie électrique à Madrid, qui en est éloigné de $76 \mathrm{~km}$. La puissance actuellement utiliséc est do $15000 \mathrm{HP}$, mais l'usine est prévue pour pouvoir en développer $25000\left(^{*}\right)$.

Un barrage a été construil immédiatement à l'aval du point où la Guadelia se jette dans le Tage. Il crée un réservoir, do i5o hectares de superficie, et de 5300 ooo $\mathrm{m}^{3}$ de capacité, qui s'élend sur les deux cours d'ean. La hauteur du mur est de $26 \mathrm{~m}$. audessus du niveau de la rivièrc ; son ćpaisseur est de $22 \mathrm{~m}$. à la base el de $6 \mathrm{~m}$. au sommct, avec profil triangulaire. Il est établi en forme de voûte trìs marquée, avec in rayon de courbure à l'extrados de $230 \mathrm{~m}$. ; sa Jongucur à la crête est de 240 mètres. Ic canal d'amenće a une longucur de $430 \mathrm{~m}$., et aboutit à la

(*) K. MEver.-Zeitschrift des Vereines deutscher Ingenielle, du co aout I 910 . 